\title{
Cost Effectiveness of Type 2 Diabetes Patients: A Research Framework
}

\author{
Suzana Sulaiman \\ Department of Professional Accounting Studies, Faculty of Accountancy, \\ Universiti Teknologi MARA, 40450 Shah Alam, Selangor, Malaysia \\ Nazreen Sahol \\ Department of Professional Accounting Studies, Faculty of Accountancy, \\ Universiti Teknologi MARA, 40450 Shah Alam, Selangor, Malaysia
}

\begin{abstract}
The growing of Metabolic Syndrome (MS) has contributed to higher risk and costs in managing Type 2 Diabetes patients. Diabetes patients are exposed to develop serious health complications. It can lead to related diseases affecting heart, eyes, kidneys, nerves and teeth. There is no proper framework on operation management and cost effectiveness in treating diabetes patients in Malaysia. Hence, it demanded on the elements and associated costs incurred by both healthcare provider and patients. This paper adopted five stages in analyzing its relevant literatures. Cost effectiveness, healthcare provider, operation management and self-management were the literatures studied. It also highlighted on management accounting gained its relevancy and contribution towards health industry. As a result, a cost effectiveness of Type 2 Diabetes framework was developed. Cost effectiveness is a key component in determining value for money. This framework provides the value for money model for Type 2 Diabetes patients. The framework clearly provides the components of cost-effectiveness and 3Es: Economy, Efficiency; Effectiveness. Objectives, resources, input, processes, output, outcomes (intended or intended) and other influences are the components.
\end{abstract}

Keywords: Type 2 Diabetes; Cost Effectiveness; Management Accounting; 3Es; Value for Money

\section{ACKNOWLEDGEMENT}

This work was supported by the MITRA Perdana Research Grant, Universiti Teknologi MARA (UiTM), Malaysia [600-IRMI/PERDANA 5/3/MITRA (008/2018)-3.

\section{INTRODUCTION}

There is no proper framework on operation management and cost effectiveness in treating diabetes patients in Malaysia. So, the objective of this paper is to develop a framework for cost effectiveness of Type 2 Diabetes patients. Cost effectiveness is an optimal use of resources in 
achieving the intended outcomes. There are many components required in achieving this objective: main and sub-components.

The objectives are supported by the research questions and problem statement. Diabetes patients can expose themselves to many infections that lead into severe illness. Without proper measurement, diabetes patients will continue on conventional treatment with insulin, not achieving healthy diet and lifestyle.

This paper adopted five stages in analyzing its relevant literatures. Literatures were identified based on cost effectiveness components. It started with literatures both on healthcare provider and operation management. Followed by exploring on the generic main processes provided by healthcare provider and understanding on self-management ability by Type 2 diabetes patients.

It is important to understand the role of management accountants, different categories of costs and value analysis before a cost effectiveness of Type 2 Diabetes patients' framework is developed.

\section{OBJECTIVES}

The main objective of this study is to develop a cost effectiveness research framework on Type 2 Diabetes Patients. The framework will assist any future research in determining the components of cost effectiveness. Cost effectiveness is the optimal use of resources to achieve the intended outcomes.

Hence, there are many sub-components in achieving such developing framework objective. This framework will provide both the main and sub-components. Each of these components will identify the associated costs in managing Type 2 Diabetes patients. Basically, the main component consists of objectives, resources, input and output of such patients. Operation management, other influences, outcomes (intended and unintended) and value improvement are the sub-components of their treatments.

This framework provides assistance to future related research in determining the ' $3 \mathrm{Es}$ ': Economy; Efficiency; Effectiveness. Understanding this framework can also be used in enhancing understanding on the concepts of 'Spending Less', 'Spending Well' and 'Spending Wisely' in treating Type 2 Diabetes patients. The framework also provides their potential costs associated to productivity and economic costs.

The paper extends to include an extended component to both the other influences and outcomes. Value improvement is an important element which the framework also considered. The value improvement is to both the healthcare provider and Type 2 diabetes patients.

\section{RESEARCH QUESTIONS}

The above developing framework objectives are supported by the following research questions:

1. What are the components in managing Type 2 Diabetes patients?

2. What are the elements and associated costs in each of these components?

3. How healthcare provider determined the ' 3 Es' in managing its Type 2 Diabetes patients?

4. What are the value improvement expected from both healthcare provider and Type 2 Diabetes patients?. 


\section{PROBLEM STATEMENT}

Metabolic syndrome (MS) is a cluster of metabolic disorders. MS is a serious health condition that affects adults and places them in higher risk cardiovascular disease, diabetes, stroke and diseases related to fatty buildups in artery walls. Diabetes is chronic disease that occurs when the pancreas is no longer able to make insulin, or when the body cannot make good use of the insulin it produces. Insulin is a hormone made by the pancreas, acts like a key to let glucose from the food to pass from the blood stream into the cells in the body to produce energy. All carbohydrate foods are broken down into glucose in the blood. Insulin helps glucose get into the cells. Not being able to produce insulin or use it effectively leads to raise glucose level in the blood (known as hyperglycaemia). Over the long-term glucose levels are associated with damage to the body and failure or various organs and tissues.

There are three main types of diabetes - type 1; type 2 and gestational. People with diabetes have an increased risk of developing a number of serious health problems. Consistently high blood glucose levels can lead to serious diseases affecting the heart and blood glucose levels can lead to serious diseases affecting the heart and blood vessels, eyes, kidneys, nerves and teeth.

People with diabetes also have a higher risk of developing infections. Diabetes is a leading cause of cardiovascular disease, blindness, kidney failure and lower limb amputation. Hence, maintaining blood glucose levels, blood pressure and cholesterol at or close to normal can help to healthy diet and lifestyle.

However, there has not been a proper framework on operation management and cost effectiveness in treating diabetes patients in Malaysia, specifically. This alternative treatment may improve diabetes patients' lifestyle and not relying on conventional treatment with insulin.

\section{RESEARCH METHODOLOGY}

This paper develops a cost effectiveness framework for Type 2 Diabetes patients. Fully relevant literature review analyses are adopted. Firstly, the analyses started with exploring both healthcare provider and its operation management. Healthcare is an industry that is different than other industries. Its diverse industry also offered numerous services.

Secondly, understanding the industry provided knowledge on the practices and processes of the services provided by healthcare. These will provide awareness on the financial resources, policies and operation management. The exploration can assist in capturing the balanced between quality of services provided and cost incurred can be identified and balanced. This stage also extended and included literature on self-management ability by Type 2 diabetes patients.

Thirdly, the methodology further included the main activities in the operation management process. Planning or designing, organizing and supervising of the assets, costs and human resources are the main activities. Understanding these activities and their costs are crucial in improving healthcare profitability.

Fourthly, this study extended its literature on the role of management accountants and different categories of costs. Financial, economic and productivity costs are part of the categories. These literatures provided examples of costs under each category. This further led into the understanding 
of value analysis. A value-based healthcare provider will adopt value analysis in continuously improving services to its patients.

Finally, based on the above literature a proposed cost effectiveness of Type 2 Diabetes patients' framework is developed. The framework can be adopted and adapted by future related research.

\section{Introduction}

\section{LITERATURE REVIEW}

Healthcare is an industry that different from all the other industries. It is an industry with many and diverse competitors. Healthcare is part of a diverse industry as it offers multiple services. Healthcare normally offered services for the diagnosis, treatment, prevention of injury, illness, disease and other physical and mental impairments.

Hence, healthcare needs proper management of its operations. Operations management that focuses on the practices designed to monitor and manage all of the processes. This includes the production and the distribution of products and services. Better operation management can further be supported by efficient self-management ability in patients with Type 2 diabetes.

Healthcare generally leads to vigorous competition in which companies win by producing the best value for customers. Certain healthcare providers survived and succeeded even with high costs but with good outcomes for customers. However, high costs are not necessarily the indicator for good outcomes. This perception needs to be changed by looking at healthcare providers that delivered the highest value for patients. Best value is when patients are rewarded with highest value at the lower costs.

\section{Operation Management}

Operation management focuses on the practices to monitor and manage all of the processes within the production and distribution of products or services. The largest activities focus on products creation and service development and the efficiency with which are distributed. It often includes analyzing an organization's internal process. Hence, the way an organization carries out operations management depends upon the nature of products or services it offers.

Operation management in healthcare is concern with the general function in running healthcare facilities. These would include looking into the financial resources, implementing policy, administering both the medical and non-medical staff. Operation management is seen as a way to increase efficiency in providing medical services to patients. Managers must work towards increasing the quality of services provided whilst trying to control the cost of delivering the services. Trained and well-paid medical and non-medical staff can ensure the achievement of quality services.

\section{Element and explanation of operation management costs}

There are three main activities in the operation management process; planning or designing, organizing and supervising of the assets, costs and human resources of the health facilities. Constant adjustment or improvement may be necessary to achieve high "profitability". 


\section{Planning and designing}

Managers will plan and design the product and process by embracing the idea of focusing on delivering the best services to the patients. Planning starts from the initial stage right up to the implementation stage. There can be several testing phases involved. During this stage sufficient consideration must be made on both the technical and non-technical aspects as well as the costs involved at each stage.

At this stage also, processes may need to be updated and new product need to be introduced to provide alternative medicine to patients.

\section{Organizing}

This will be the base of maximizing efficiency of the health facilities. Management of resources especially human resource is very crucial and therefore due care is taken by managers when assigning tasks to individuals and arrange the tasks according to the organizational structure. Costs of these resources will be collected to be able to measure the effectiveness on treating the patients. Raw materials and facilities need to be carefully matched with the human and processes to avoid unnecessary costs and inefficiencies.

\section{Supervising}

Managers will give detail and specific instructions of the tasks to the staff via tasks scheduling. Proper monitoring will be conducted to ensure that jobs are performed and materials consumed accordingly. There is always room for improvement for example introducing new way of treating patients that is cost effective.

\section{Purpose for effective Operation Management Costs}

One of the major global challenges in the healthcare facilities is the rising cost. The main contributor to this trend, are aging populations and medical technology. However, most healthcare providers are unable to properly measure and manage the true costs and value services provided. It is important for healthcare providers to find better ways to measure and manage healthcare costs and value to achieve improved outcomes and lower costs.

Cost control at healthcare is crucial and one of the first areas of focus for operations manager. Continuous high costs will create burden on taxpayers, health insurance holders and healthcare organisations themselves. Hence, striking a balance between treatment provided and costs is very crucial. This is where patients are provided with the best value treatment.

Efficient cost control and value analysis are needed. Inefficiently cost cutting on budgets, limiting technology and purchased equipment may affect the level and quality of services provided. So it is the role of the managers to streamline between costs and the quality of services offered. The bottom-line is for the operations management to play a responsible role to oversight the healthcare facility operations. Ensuring its function efficiently, capable to provide adequate and reliable treatment to the community it serves.

\section{VALUE ANALYSIS (VA)}

Value Analysis is a systematic and organized approach to provide the necessary functions in a product or service at the lowest cost. It promotes the substitution of materials and methods with 
less expensive alternatives, without sacrificing functionality. It is focused solely on the functions of various components and materials, rather than their physical attributes. It is also led as value analysis.

(www.jstor.org/stable/43620667?seq=1). Mr. Lawrence D.Miles, an engineer at General Electric Company has developed the concept of Value Engineering (VE). He created this technique in response to critical material shortages during World War II. Mr. Miles recognized that proper analysis of a product's function often led to improved performance and cost savings as alternative materials or manufacturing methods were discovered and employed. This formal, organized study of functions to satisfy the user's needs at the lowest life cycle costs through applied creativity has saved money and/or improved quality for companies and government ever since.

VA can be adopted in various was to achieve product, service or process cost savings. There is a need to decide on the critical manufacturing area, whether on the new product or service development or items currently in production. Figure 1 provides the typical VA methodology framework and it is as follows:

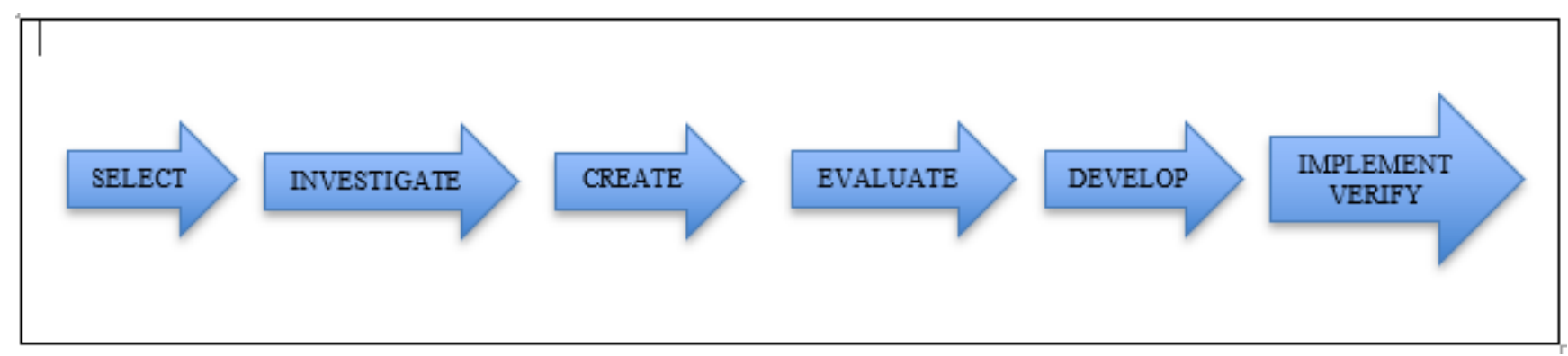

Figure 1: VA Methodology Framework

The advantages of VA include the following:

\section{Eliminating Costs}

A critical advantage to using VA is its potential for reducing costs, which is a benefit that permeates all advantages of the system. Because VA breaks down a product or service into components, it enables to analyze each component on its own, evaluating its importance and efficiency. VA analysis correctly implemented and applied allows to identify components that are not worth the cost required and that can be eliminated or replaced with an alternative. In this manner, the process for the product or service being analyzed is refined to be done at less expense.

\section{Modernizing}

The VA process often allows users to root out practices that have grown out of date and can be replaced with more modern approaches. This is particularly beneficial when something has been done the same way for an extended period of time. Because the old way works and was new when it was instituted, it had little impetus to make changes. However, a VA, which is for questioning every step of a process, can reveal new methods that are cheaper, more efficient and sometimes more effective. 


\section{Design Flaws}

Value analysis can uncover design flaws that not only operate inefficiently but also create problems. In the case of a product, this could mean a high rate of malfunctioning items, creating customer complaints and warranty claims that put a strain on personnel and inventory. It also can lead to bad publicity and damage to the product brand and the company producing it. Similarly, in the case of a service, value analysis can help pinpoint design flaws in the customer support system that causes service to fall short of customer expectations.

\section{Customer Service}

VA is oriented to weigh costs and the benefit to customers of a product or service. It forces to consider every aspect of a process in the context of how it serves the customer, which could be a consumer or another business. This means that each step in the process is scrutinized and questioned from the perspective of the benefit that it provides the customer. If the benefit to the customer is small and the step is not necessary for the product or service as a whole, it can be eliminated, allowing you to streamline your operation and to reduce the use of resources.

The output of VA is a series of improvement plans that raise the value of the target product or service. VA emphasizes on the functionality and meeting customer requirements within the allowable cost parameters. VA goes beyond the particular styles or configurations of current products or services to consider the functions that lie at the heart of the product or service in order to come up with innovative ways to achieve desired functionality with less cost or effort. VA is a generally critical success management accounting tool specifically towards Target Costing implementation. Healthcare provider needs to change their strategies to market-driven philosophy, price-down, cost-down and value-based which allow them to win considerable share of their respective markets.

\section{VALUE-BASED HEALTH PROVIDER}

Value-based health provider explores ways to improve value for patients, improving processes and reducing costs without sacrificing outcomes. This dismisses traditional cost-cutting initiatives that can actually lead to higher costs and lower-quality care. Previously, healthcare providers used methods such as the ratio-of-costs-to-charges (RCC) or costing based on relative value units (RVUs) in developing for their pricing (private healthcare providers). They only cost services that can be cost, while the non-reimbursed costs are arbitrary allocated as overhead pools. Departments and procedures are allocated with these costs but not to all the other services centers that also involved in treating medication over the patient's entire treatment cycle.

Since there is no detail on each cost activities, management will normally rely on the line-item from the income statement. These items are by categories (employees, equipment, devices, imaging, laboratory tests, pharmaceuticals) and not by medical condition. Common practice in costs cutting will normally be on the line-item. For example, 5\% costs cutting across the board in personnel costs. Patients, outcomes could be harmed with this arbitrary cost cutting.

Value is improved as better patient outcomes relative to the costs of achieving them. Value improvement is the main goal of any healthcare provider and also needs to identify appropriate measures. Healthcare providers are expected to be accountable for their patients' outcomes. 
However, most healthcare providers have overly focused on introducing checklists and measuring compliance with evidence-based rather than achieving better patient outcomes. Compliance activities are important but it is backward-looking and therefore inadequate. Value improvement is forward-looking and drive toward transformational and therefore a more powerful exercise.

Appropriate measurements are required in measuring health outcomes. The measurements that able to show improve in the outcomes. Outcomes can be measured as improve, better or faster due to new technique (medical device, drugs or surgical). Improving survival rates, increasing in delivering primary and preventative care for patient could also be considered as part of the outcome measurements. Functional status (improved ability, more competent, no infections, no readmissions, etc) of patients after treatment can also be considered as one of the measurements too. These measurements can be adopted in distinguishing between high or low performing healthcare providers.

Great emphasis on value-based by adopting relevant measurements will able healthcare provider to track and report outcomes. This will lead to much better performance and much lower variability in outcomes. Healthcare providers with this focus will have great professional pride and commitment to their patients. It will also be a motivator for healthcare providers to improve their outcomes gradually.

Value-based with relevant measurement will also assist in achieving sustainable cost reduction. As is provides understanding towards the structure of delivery. It looks at how a healthcare provider spending money to treat patients for specific medical conditions. This process will allow inefficient and ineffective processes to be understood and identified. Proper fundamental improvements are possible which enable fewer or less expensive resources, same amount of work or better, without effecting outcomes. This will lead into sustainable and justified for any cost reduction decisions.

\section{SELF-MANAGEMENT ABILITY IN PATIENTS WITH TYPE 2 DIABETES}

Since diabetes is a chronic disease that requires a patient to make a multitude of daily selfmanagement decisions and to perform complex care activities. Diabetes self-management education is the process of facilitating the knowledge, skill and ability necessary for diabetes selfcare. Health provider can provide self-management support in implementing and sustaining coping skills and behavior needed to self-manage on an ongoing.

It is important for health care providers to include settings in their resources to support these diabetes patients. This is to ensure the patients receive consistent and on-going both Diabetes selfmanagement education (DSME) and Diabetes self-management support (DSMS).

Both DSME and DSMS programs should designed to address the patient's health beliefs, cultural needs, current knowledge, physical limitations, emotional concerns, family support, financial status, medical history, health literacy and other factors that influence each patient's ability to meet the challenges of self-management.

Proper DSME and DSMS will contribute benefit toward both cost effectiveness and value analysis. These two can be reflected by the reduction in both hospital admissions and readmissions. Estimated lifetime health care costs will be reduced with lower risk for complications too. DSME 
and DSMS can also provide positive effect on other clinical psychosocial and behavioral aspects of diabetes patients [1].

DSME and DSMS also being reported to reduce the onset and/or advancement of diabetes complications, improve quality of life and lifestyle behavior. These include exposing patients to a more healthful eating pattern, regular physical activities, enhance self-efficacy, healthy coping, decrease diabetes-related distress and depression. This clearly provides value-added benefits to diabetes patients.

Expectations played major driver of diabetes patient behavior. Their expectations towards the future of current diabetes medications, the need for additional treatments and possibility of remission (not needing medications) in the future will be their main goals [2]. According to this study, majority or the patients expected that they would be able to discontinue their diabetes and hypertension medication in about 5 years. Nearly all expected not needing additional diabetes medications and majority thought that their conditions could be cured.

Diabetes patients' expectations were closely related to how certain they were that they could achieve significant lifestyle changes. While others who were uncertain that they could stop medications in the future were more likely to mention the influence or external factors such as the opinions of their doctors, scientific advancements, life stressors and family history. The duration of taking the medication is also part of the expectation in managing such disease.

Health provider costs will be unable to be reduced unless incidence rates and diabetes-related complications are reduced. Hence, health providers are expected to better understand the educational process and expectations for both DSME and DSMS. This can then reflected by their abilities to integrate them into their routine are and achieve their ultimate goals of more engaging and informed diabetes patients.

\section{MANAGEMENT ACCOUNTANTS ROLES}

Healthcare has been seen as 'fact-free' zone with missing incomplete or distorted measurements of performance. Management accountants can play a championing role to assist costs analysis and value measurements. New measurements to focus on promoting value creation within health providers' industry.

Management accountants should championing measurements that able to produce more tangible value improvements, quantified outcomes and lower costs. Better measurements will improve value for patients, improve processes and reduce costs without sacrificing outcomes.

This is to avoid overly focused on introducing checklists and measuring compliance with evidencebased protocols. Healthcare should be focusing more on achieving better patient outcomes. Hence, measuring processes is crucial and important in moving forward towards transformational value improvement within healthcare providers.

\section{Management Accounting and Healthcare Providers}

Management Accounting is not only adopted by profit-oriented organisations but can also be extended to non-profit organisations. For example, a group of researchers from Kaplan also 
suggested for the management accounting improvement in the healthcare industry. The application of a well-established management accounting technique that can increase the value of care delivery at the lowest possible cost. For example, the concept of time-driven activity-based costing (TDABC) that was adopted by manufacturing, retail and services is perfectly now for healthcare. This concept can help clinicians to understand the cost of treatment and see how to reduce costs while maintaining and often increasing care quality. He also suggested for the healthcare leaders to create a central "value management office" to enhance the institution's ability to improve outcomes and reduce costs. Since healthcare provider is a 'fact-free' zone, an amazing sector for management accountants, with experience and expertise in costing to make important contributions.

This further supports the claim that management accounting has gained its relevancy through its extension into other industries which include non-manufacturing organisations which to include adopted by health industry. Management accounting tools are internal usage and not compulsory, hence organisations adopted the best practices that best suit their organisations at a certain particular situation. In other words, management accounting tools are adopted and tailored to need of the respective organisations. The following paragraphs will briefly explained common terms used by management accounting in healthcare.

\section{COSTS}

Costs are those expenses incurred in producing or rendering a product or service. Cost can generally be classified into their main category such as material, labour and overhead. Overhead are those costs that are indirectly incurred in producing and rendering the product or service, respectively. Costs can also be classified into product and period cost. Product costs are costs that are necessary and integral part of producing the finished product. Period costs are costs that are matched with the revenue of a specific time period rather that include as part of the cost of a salable product [5].

\section{Cost-Benefit Analysis (CBA)}

Cost-benefit analysis is the exercise of evaluating an action's consequences by weighing the pluses, or benefits, against the minuses, or costs. It is the fundamental assessment behind virtually every business decision, due to the simple fact that business managers do not want to spend money unless the resulting benefits are expected to exceed the costs. As companies increasingly seek to cut costs and improve productivity, cost-benefit analysis has become a valuable tool for evaluating a wide range of business opportunities, such as major purchases, organizational changes, and expansions.

Some examples of the types of business decisions that may be facilitated by cost-benefit analysis include whether or not to add employees, introduce a new technology, purchase equipment, change vendors, implement new procedures, and remodel or relocate facilities. In evaluating such opportunities, managers can justify their decisions by applying cost-benefit analysis. This type of analysis can identify the hard dollar savings (actual, quantitative savings), soft dollar savings (less tangible, qualitative savings, as in management time or facility space), and cost avoidance (the elimination of a future cost, like overtime or equipment leasing) associated with the opportunity. 
A formal cost-benefit analysis is a multi-step process which includes a preliminary survey, a feasibility study, and a final report. At the conclusion of each step, the party responsible for performing the analysis can decide whether continuing on to the next step is warranted. The preliminary survey is an initial evaluation that involves gathering information on both the opportunity and the existing situation. The feasibility study involves completing the information gathering as needed and evaluating the data to gauge the short- and long-term impact of the opportunity. Finally, the formal cost-benefit analysis report should provide decision makers with all the pertinent information they need to take appropriate action on the opportunity. It should include an executive summary and introduction; information about the scope, purpose, and methodology of the study; recommendations, along with factual justification; and factors concerning implementation.

Capital budgeting has at its core the tool of cost-benefit analysis; it merely extends the basic form into a multi- period analysis, with consideration of the time value of money. In this context, a new product, venture, or investment is evaluated on a start-to-finish basis, with care taken to capture all the impacts on the company, both cost and benefits. When these inputs and outputs are quantified by year, they can then be discounted to present value to determine the net present value of the opportunity at the time of the decision.

Cost-benefit analysis is often used in the field of health services, where it may be inappropriate to monetize health effect. Typically the cost-benefit analysis is expressed in terms of a ratio where the denominator is a gain in health from a measure (years of life, premature births averted, sight-years gained) and the numerator is the cost associated with the health gain. The most commonly used outcome measure is quality-adjusted life years (QALY).

\section{Financial cost}

Financial costs represent actual expenditure on goods and services purchased for any project such as in the diabetes treatment activities. Project costs were classified as capital and recurrent costs. Capital costs include initial cost on buildings, equipment and starting up training. Financial equipment costs are calculated using straight-line depreciation and the item cost is divided by the expected life of the item. Startup activities include initial project training sessions for staff and development.

Recurrent relate to continuous costs incurred in running the identified projects. These include staffing, training, building maintenance and mass media cost. Financial personnel costs include the cost paid by the project for each staff member and to include honorarium payment. Data on these costs can be obtained from project documents, interviews and observations. Figure 2 summarises the components of financial costs.

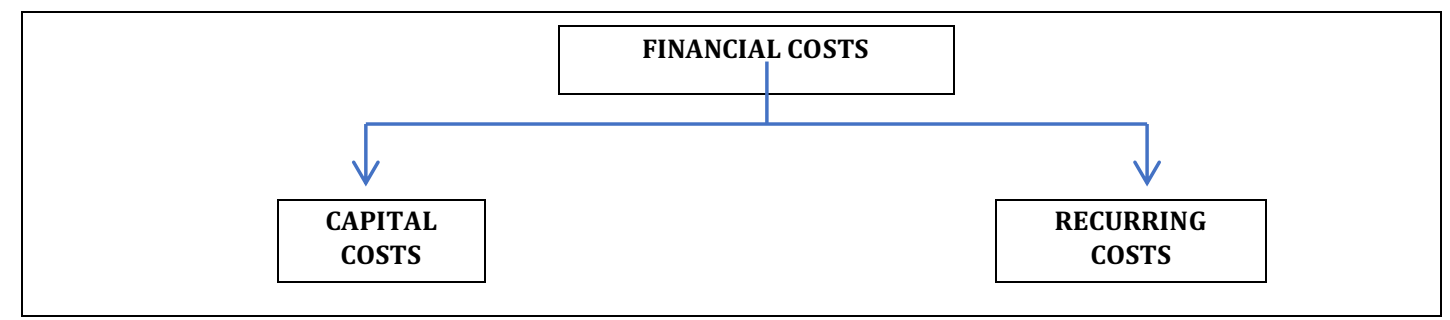

Figure 2: Financial Costs Framework 


\section{Economic cost}

Economic costs include the estimated value of goods or services for which there are no financial transactions or the price of the good does not reflect the cost of using it elsewhere. It is an opportunity cost or notional costs of the inputs. These costs include free of charge buildings or area used by the diabetes treatment activities. The economic cost of this space is calculated based on the square footage used and the prevailing market rental rates for the space. The economic costs for volunteer staff should reflect the full notional salaries by multiplied their time allocation of the project and relevant rate. Economic costs also include a market evaluation of any donated items. Economic costs for free educational radio air-time in transmitting mass media messages need also to be identified. These costs can be estimated from interviews with healthcare provider, project coordinator and observation of the resources used.

In summary both financial and economic costs are summarized by [4] as,

"The major difference between financial and economic costs was the inclusion of the full cost of employed staff, the market value for donated and discounted items, and the rental value for donated building space. The financial cost includes only honorarium payments for many staff, which the NGO made. The economic costs include a valuation of the staff time actually put into the project by these honorarium staff". Figure 3 provides the economic framework.

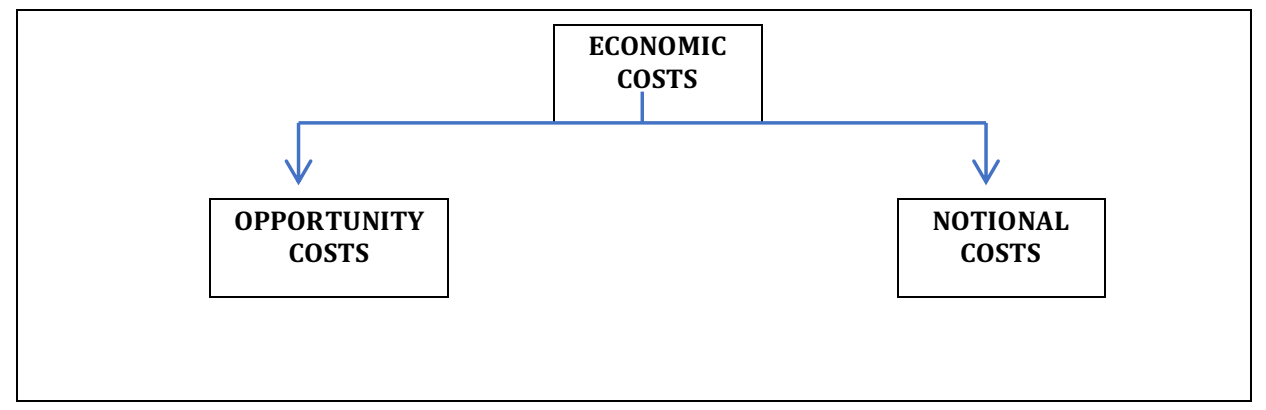

Figure 3: Economic Framework

\section{Productivity Costs}

The term productivity costs has been defined by the US Panel on Cost-Effectiveness Health and Medicine as

"the costs associated with lost or impaired ability to work or engage in leisure activities due to morbidity and lost economic productivity due to death"

The productivity cost also consists of both financial and non-financial. The mortality includes lost economic productivity such as premature death and other indirect costs [3]. For example, some of the indirect costs that caused the economic productivity pertaining to harm reduction programs included cost incurred relating to rehabilitation centers, jurisdiction process, potential corruption, retain and prison centers and increase in security costs. Figure 4 below shows the main components of productivity costs. 




Figure 4: Productivity Framework

\section{VALUE FOR MONEY}

A key component determining value for money, also known as cost-effectiveness. The following figure provides the value for money model. This model provides the components within the value for money. The main components of value for money consist of: Objectives; Resources; Inputs; Processes; Outputs; Other Influences; and Outcomes. It is an outcome to measure on how much resources were allocated to achievements. Cost-effectiveness measurement consist of the 3Es: Economy; Efficiency; Effectiveness.

Economy is minimising the cost of resources used or required inputs (feeder). In other words, economy is the ability to spend less. This is reflected in the following Figure 5.

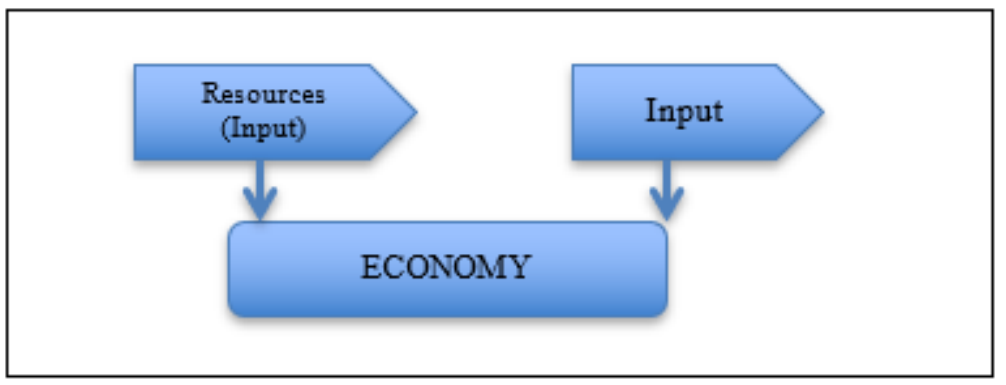

Figure 5: Economy Model

Efficiency is the relationship between the resources or input to produce and the output (results). This will reflect on whether resources have been well spent. It is captured by Figure 6.

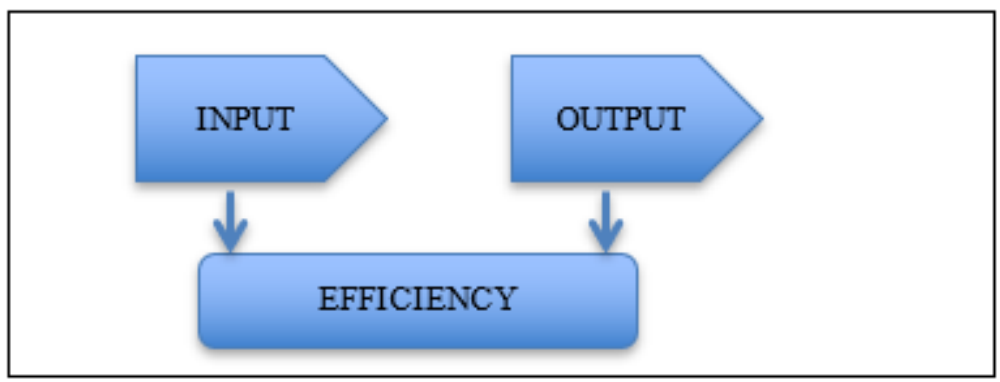

Figure 6: Efficiency Model 
While effectiveness is the relationship between the actual results and the intended outcomes (objectives). This measurement will look into whether the spending has been wisely spent. Figure 7 summarises and the relationship.

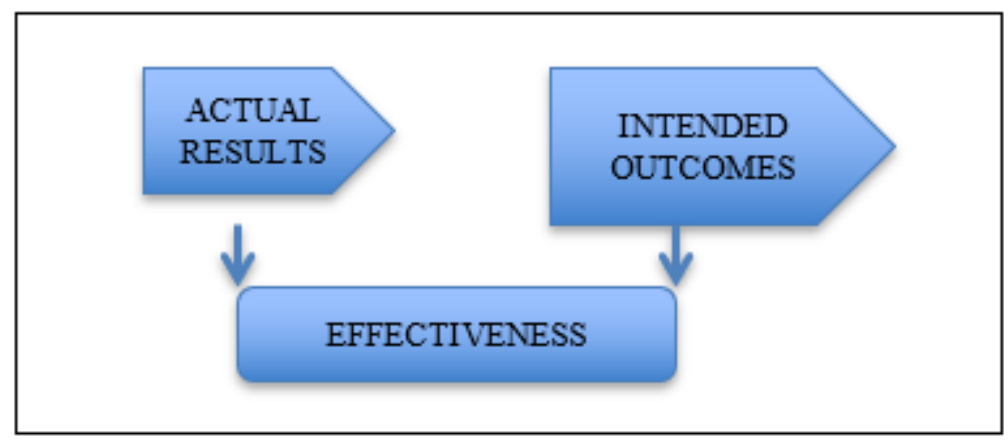

Figure 7: Effectiveness Model

The 3Es can be later extended to link the inputs (feeder) and intended outcomes (objectives). This is called as cost-effectiveness. It is the optimal use of input or resources to achieve the intended outcomes. Cost effectiveness is also part of value for money as it incorporated all the above mention main components.

Figure 8 provides all the 3Es' main components and their relationships and cost effectiveness. This model started with determining the objectives and the resources required. This is followed by the inputs required by the identified processes in producing the outputs. The output later will be matched against the outcomes. Influenced by other factors (expected or unexpected) may result into both intended and unintended outcomes.

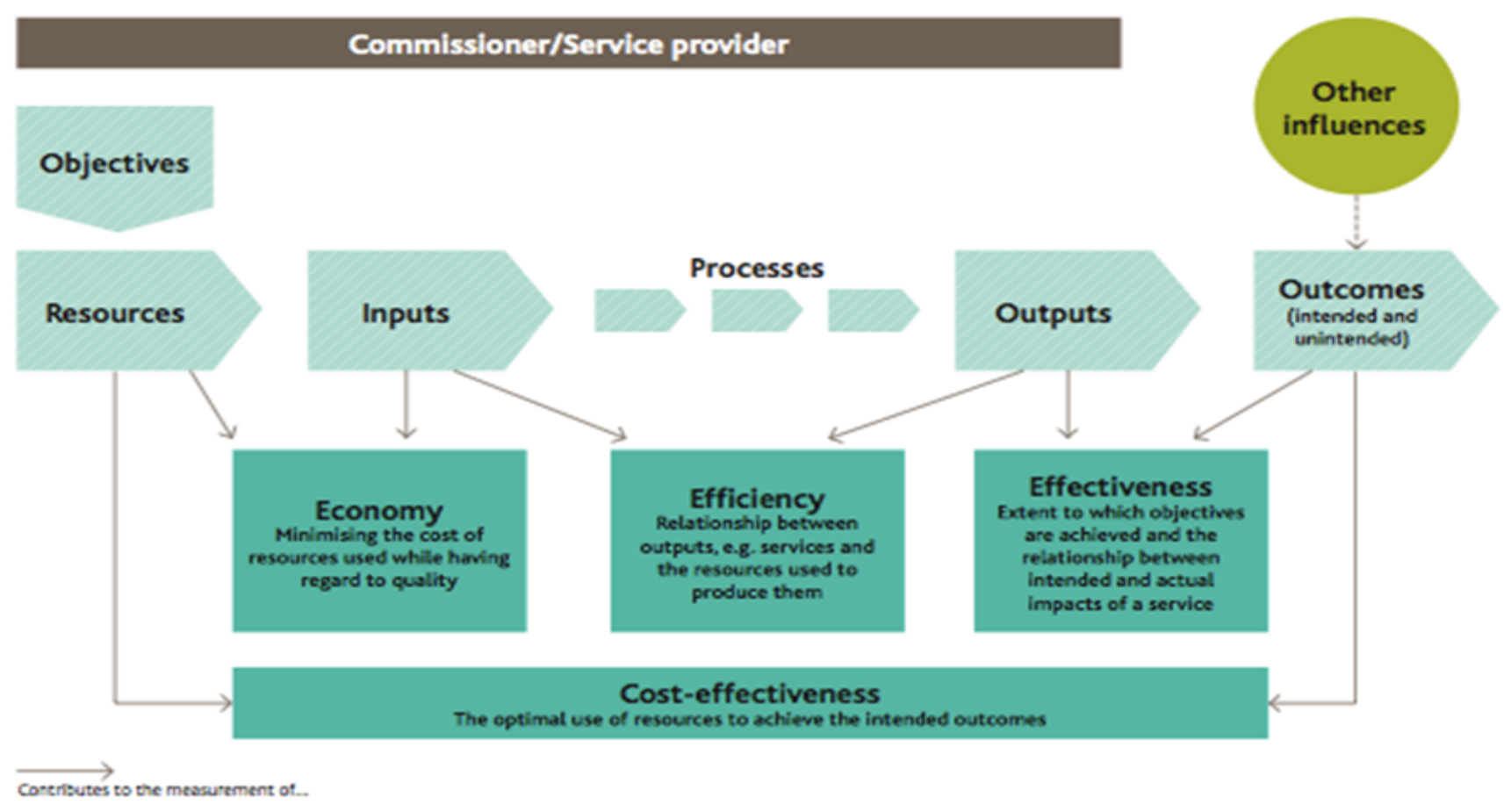

Figure 8: Value for Money Model 


\section{CONCLUSION}

This section will combine all the above explained components and models into a framework. Figure 8 provides the cost effectiveness framework for Type 2 Diabetes patients. The explanation will start from the left of the entire value chain. Initially, objectives need to be identified and agreed upon. Resources availability is recognized before transfer as input into the entire process.

Both the resources availability and input will lead into measuring the first 3Es, Economy. It is the cost of resources used at its minimal or 'spending less'. Costs can be mainly grouped into productivity and economic costs. Financial costs, non-financial costs, opportunity costs and notional costs are their sub-components.

The operation management process involved two parties: the healthcare provider and Type 2 patients themselves. Healthcare providers are expected to conduct planning and designing, organizing and supervising their entire activities. While Type 2 Diabetes patients are expected to be able to continuously self-manage and enjoy quality of life. 'Spending well' is achieved by properly manage this process operation management.

The process is followed by the output from the operation management process. Effectiveness from the entire process is measured by its outcomes. Outcomes can be intended or intended. Besides the operation management process, both outcomes can also be the result of other influences. Intended outcomes are as expected and listed in the objectives. On the other hand, the unintended outcomes are those unexpected and not part of the identified objectives. They can be in the formed of both positive and negative outcomes.

The outcomes from the entire process should create value to both the healthcare providers and Type 2 Diabetes patients. Success of the entire operation management process relies on the values provided to both the healthcare provider and Type 2 Diabetes patients. A successful healthcare provider is its ability to provide consistent and on-going self-management support to Type 2 Diabetes patients.

While, Type 2 Diabetes patients will gain from this operation management if they could achieve significant lifestyle changes. Stop medications in the future and reduction in diabetes-related complications are few values expected from Type 2 Diabetes patients from this operation management process. 'Spending wisely' is achieved if the resources are effectively utilized and the expected values are accomplished. 


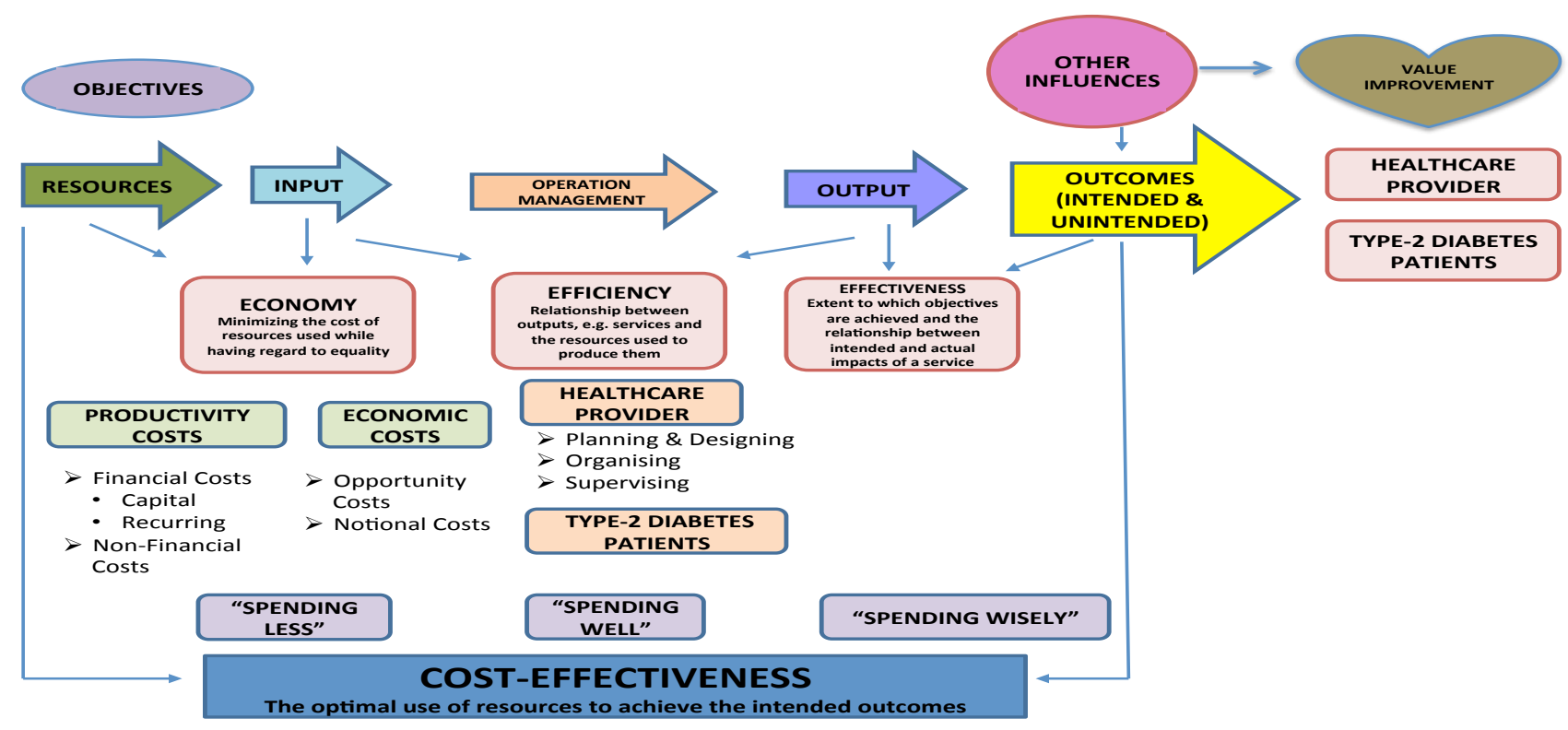

Figure 9: Cost Effectiveness for Type 2 Diabetes Patients Framework

The above Figure 9's framework can be adopted and adapted by any future cost effectiveness studies, both medical and non-medical. Researchers can substitute the sub-components based on the objectives, those involve in the process and the end-users.

\section{References}

[1]. Margaret A. P., Joan, B., Marjorie, C., Paulina, D., Martha, M. F., Amy, H. F., Melinda, D. M., Linda, S., \& Eva, V. (2015). Diabetes Self-management Education and Support in Type 2 Diabetes: A Joint Position Statement of the American Diabetes Association, the American Association of Diabetes Educators, and the Academy of Nutrition and Dietetics. Diabetes Care 2015 Jul. 38(7): 1372-1382

[2]. Paige, C. F., Aviva, G. N., Michael, Q., Elbert, S. H., \& Neda, L. (2017). Patients' Future Expectations for Diabetes and Hypertension Treatments: Through the Diet... I Think This is Going to Go Away. Journal of General International Medicine, 32(1): 49-55

[3]. Sendi, P., Schellenberg, F., Ungsedhapand, C., Kaufman, G., Bucher, H., Weber, R., Battegay, M. and Swiss HIV Cohort Study (2004). Productivity Costs and Determinants of Productivity in HIV-Infected Patients. Clinical Therapeutics, Vol. 26, No. 5, p. $791-800$

[4]. Vickerman, P., Kumaranayake, L., Balakireva, O., Guinness, G., Artyukh, O., Semikop, T., Yaremenko, O. \& Watts, C. (2006). The Cost-Effectiveness of Expanding Ham Reduction Activities for Injecting Drug Users in Odessa, Ukraine. Sexually Trasmitted Diseases. Vol. 33, No. 10, p.S89-102

[5]. Weygandt, J., Kimmel, P. \&Kieso, D. (2008). Managerial Accounting, John Wiley \& Son Yin, R. K. (1994). Case Study Research: Designs and Methods. Newberry Park, CA: Sage Publications. 